\title{
Purebred dogs and canine wellbeing
}

\section{Introduction}

Conscientious breeders of purebred dogs usually have several conflicting goals that they attempt to achieve. ${ }^{1}$ They might, for instance, want to breed dogs useful for performing various tasks, that can succeed in conformation shows, conform to the breed standard, make their owners happy and are happy themselves. These goals might sometimes conflict, meaning breeders have to prioritize. Likewise, kennel clubs will have to decide which goals are most important when setting up their policies and rules, and legislating bodies will have to prioritize when imposing legal restrictions on breeders of dogs. However, I will not, in this article, discuss how one ought to prioritize. Rather, I will do some groundwork in order to make a more rational prioritization possible. If we first determine what we ought to do given each goal taken separately, we will be able to clearly see where the conflicts between different goals lie, and move on to a discussion of how to prioritize when they conflict.

In this article, I will investigate what we ought to do given the goal of canine wellbeing and point out where this goal might come into conflict with other goals that we have. It is usually assumed, by breeders considering themselves conscientious and concerned with animal welfare, that a breeder fulfils her canine wellbeing-related obligations if she tries to breed as mentally and physically healthy dogs as possible within her breed of choice. I will argue that this breeder common sense morality cannot be justified; canine wellbeing calls for radical reforms. Many dog breeds ought not to be improved but outright abandoned. However, some kind of breed system can still be justified by canine wellbeing considerations; altering rather than completely abandoning the praxis of dividing dogs into breeds would be optimal for preserving genetic diversity, which in turn has beneficial health effects in the long run.

I will assume, for the purpose of this article, that breeding animals is not always morally wrong. I think this is a view that can be defended, not only from a consequentialist moral view, but also from a deontological animal rights view. I think Richard Haynes' moral principle of animal breeding can be incorporated into a strict animal rights view. He argues that it is morally permissible to breed on companion animals for the reason that one values the social bonding with them, as long as one has the knowledge and resources necessary to provide the offspring with rich and flourishing lives. (Haynes also thinks that morally

\footnotetext{
${ }^{1}$ A lot of what is said in this article could be generalized to the breeding of other companion animals. I will, however, focus solely on dogs throughout.
} 
permissible human procreation requires the same conditions. Haynes 2008 pp 144-145.)

32 However, in this article I will not argue for but simply assume that it is not always morally wrong to breed dogs, and that it may further be right to have various goals for one's breeding program besides producing new companions to bond with.

Since I do not discuss what the correct priorities between different goals are in this article, I use 'ought', 'reason' and other action-guiding words in their pro tanto sense, ${ }^{2}$ allowing for the possibility that they might be overridden, in the final analysis, by stronger 'ought':s or reasons. I will also use the term wellbeing undefined; I will merely assume that being in pain as well as experiencing negative emotions such as fear and frustration detracts from wellbeing. It is thus possible to breed for wellbeing, since we can strive to avoid deformities, diseases and mental problems. Finally, when I talk about a dog breed in this article, unless otherwise specified I refer not to a type of dog, but to a population registered under a common breed name in the kennel clubs of various countries.

\section{Breeder common sense morality}

The cynic, having watched various TV documentaries featuring purebred dogs with extreme body shapes and suffering from various illnesses, might claim that breeders of purebred dogs care nothing for their wellbeing. However, if we look at small-scale breeders rather than the puppy mill kind, my experience is that they usually do express a concern for their dogs' wellbeing. They may still end up producing lots of sickly dogs though - either because they consciously prioritize some other breeding goal (such as winning conformation shows) above wellbeing when various goals conflict, or because they simply deny that various deformities or illnesses have a negative impact on wellbeing. Likewise, my impression is that puppy buyers in general do not want a suffering dog, but may be profoundly ignorant (sometimes wilfully so) - for instance, they may argue that a dog's breathing difficulties does not negatively effect her wellbeing since she is such a calm dog and does not want to run around anyway. People's ability to delude themselves when acting immorally is of course a big problem in many contexts, not just dog-breeding, and I have no simple solution to offer. I will simply note, with regret, that this is often the case, and then put this problem aside.

I will go as far as claiming that most small-scale dog breeders who consider themselves conscientious adhere to a moral principle which we might call 'the dog breeder common sense morality principle', or BCM for short.

\footnotetext{
${ }^{2}$ Or 'prima facie', as W D Ross put it. I prefer 'pro tanto', since the term 'prima facie' sometimes means 'merely apparent'.
} 
BCM: It is morally permitted to choose any breed you want to out of the ones recognized by the kennel clubs; once this choice is made, you ought to try to produce dogs with as high a level of wellbeing as possible.

"Try to" is there because there are no guarantees in breeding. It is commonly recognized that the best-laid plans might come to naught. (The "try to" is supposed to include that one actually keeps oneself informed about relevant research, though.) The entire moral principle is a pro tanto one; it allows for other considerations, not directly related to canine wellbeing, to override it. Perhaps you can have a duty to choose a certain breed if you are in a unique position to save this particular breed from extinction. Perhaps you ought to breed for certain traditional traits even if they lessen the wellbeing of the dogs, since tradition is important. (I am not saying these are plausible views, only that BCM is a pro tanto principle and therefore allows for them. You can probably find some dog people who believe these things.) As long as we focus on canine wellbeing however, it is fairly widely accepted that following BCM means fulfilling your moral obligations. BCM implies that breeding on dogs who are known or strongly suspected to carry genes for certain health or mental problems, when there are other dogs in the same breed without these genes, at least requires justification from other breeding goals; but choosing a breed with many serious health problems need not be defended.

Obviously I do not mean to say that breeders consciously consider this principle, or that any kennel club has it written down somewhere in its policy in precisely this form. However, it is often implicitly invoked both in discussions between dog breeders and in documents, statements and policies made by the more ambitious kennel clubs. The Swedish Kennel Club, for instance, argues that we ought to counteract trends that favour exterior or mental exaggerations in dogs, for the sake of the dogs themselves, but this "counteracting" is not supposed to include the actual abandonment of exaggerated breeds, only the choosing of less exaggerated specimens for breeding within these breeds (The Swedish Kennel Club, SKK, 2012).

However, although BCM is a widely accepted principle, it can ultimately not be justified. It is not plausible that it is, from the point of view of canine wellbeing, morally permissible to choose any breed one likes. 


\section{The non-identity problem}

One way of justifying the moral permissibility of choosing any breed, even a very sickly one, would be to bring up the so-called non-identity problem. It is of course wrong to harm dogs. However, if I choose to breed dogs of a very sickly breed, no one is harmed. The puppies I produce may have various health problems, but if I had not bred on the animals I did breed on, these puppies would not have existed at all. Even if we grant, for the sake of discussion, that some lives are so bad for the individuals living them that it would have been better for those individuals never to have been born, this will most likely not be the case with the dogs I produce in my kennel. If they are not so bad off that they ought to be put down immediately after birth for their own sake, if we think that their lives (at least up to a point) will be worth living, it is hard to argue that it would have been better for them if they had never been born.

However, if it is morally permissible to knowingly produce sick individuals as long as they have lives worth living, I have no obligation to make the best I can within my breed of choice. It would be perfectly morally permissible for me to choose sicker over less sick breeding animals, as long as I can be fairly certain of producing puppies with a life worth living. Only the first part of BCM, the permissibility to choose any breed one likes, would be true. This is simply incredibly implausible and counter-intuitive. And in the philosophical literature, a large number of philosophers from various philosophical schools have argued that we do have a pro tanto duty to produce as healthy or happy individuals as we can.

\section{Maximizing wellbeing}

In addition to a duty not to create dogs with a life not worth living, we may have a duty to create dogs with as good a life as possible. If we can choose between creating a litter of dogs that will probably have a low level of wellbeing, and another litter expected to have a high level, is it not obvious that we ought to choose to bring the latter into existence? Most conscientious breeders would spontaneously answer "yes" to this question, at least if we remember that we are discussing pro tanto 'ought':s here, that could in principle be overridden by reasons relating to other breeding goals. Everything else being equal, we ought to create the litter that is expected to have a higher level of wellbeing - this much seems intuitively obvious. That we ought to maximize wellbeing or minimize suffering in future generations is also a view a number of philosophers in population ethics have supported. All utilitarians, for instance (at least when we consider same number cases - I will, somewhat simplified, assume that the number of dogs we ought to produce is set by the number of potential good homes there are, and that the question of whether to produce 100 dogs with 
wellbeing level 50 or 50 dogs with wellbeing level 100 therefore does not arise), agree that this is the case, since we always ought to maximize utility. Non-utilitarians may argue that respect for someone's autonomy is far more important than happiness when it comes to actual individuals, but still agree that we ought to, in some sense of wellbeing, maximize the wellbeing of future generations who cannot (for obvious reasons) decide for themselves. Erik Malmquist, for instance, has a basically Aristotelian approach where respect for autonomy plays a large part, but argues that in the case of future generations we ought to minimize the kinds of diseases and disabilities that limit the quality of life for any person, regardless of her particular preferences (Malmquist, 2008. p 171). There is much disagreement when it comes to whether we ought to merely minimize illnesses and disabilities or whether we also ought to enhance the capacities of future generations; Julian Savulescu argues alongside utilitarians that we ought to produce the best possible children (Savulescu, 2001, p 415), while the aforementioned Malmquist as well as Elizabeth Harman merely argues for illness and disability minimization (Harman, pp 93 and 102). Buchanan et al (2000, p 249) and James Woodward (1986) argue that it is wrong to create individuals that fall below a certain threshold - although when all the details are spelled out they tend towards illness and disability minimization as well. The debate between happiness maximizers and illness and disability minimizers may be crucial to questions of genetic enhancement of human beings, but in the current dog breeding discussions the two views would tend towards the same policy - breeding for as physically and mentally healthy dogs as possible. However, if this is the case, the first part of BCM must be false. If we ought to breed as healthy dogs as possible, this is relevant for breed choice as well, not just for the breeding decisions we make within a breed.

All the above-mentioned philosophers have, of course, discussed human procreation rather than dog breeding. However, that is of no consequence here. If dogs, as many suppose, have a lower moral status than human beings, this merely means that pro tanto reasons that stem from canine wellbeing might be outweighed by other factors that would not suffice to outweigh corresponding human wellbeing pro tanto reasons, but the difference in moral status would make no difference to the pro tanto reasons themselves. The common pre-theoretical intuition that we ought to produce as much wellbeing or as little illness and disability as possible, which a large number of philosophers have shown can be defended from a variety of moral-philosophical standpoints, is as relevant for dogs as for humans. It is the counter arguments that are specifically human. 


\section{Against maximizing wellbeing}

160 The widespread philosophical idea that we ought to minimize disabilities and diseases and/or maximize wellbeing in future generations has not gone unchallenged. Firstly, it has been argued that what is commonly thought of as disabilities should rather be seen as different abilities, and need not make the individual any worse off (Edwards, 2001); secondly, that attempting to create abled rather than disabled individuals will promote prejudices and discrimination against the disabled, thus decreasing their wellbeing (Magnus Reindal, 2000). Now, if there is nothing wrong with creating disabled humans, it seems as if there would not be any canine wellbeing related moral reasons against creating disabled dogs either. If one could only wrong the puppy buyers by producing disabled dogs, doing so might be all-thingsconsidered morally permitted if the puppy buyers know about the puppies' health problems and still choose to purchase them.

I think the first argument against preventing disabilities is stronger than many philosophers want to admit as long as we discuss human beings, but it is actually not relevant for dogs. Take, for instance, deaf people who argue that their inability to hear gives them access to a unique culture of sign language and a unique way of experiencing the world, and therefore see themselves as differently abled rather than disabled. I think many philosophers tend to dismiss this view too quickly. I do not doubt that I would be disabled if I suddenly lost my hearing,

177 but being born deaf is a different matter. I might learn sign language and not regard this as 178 very special, but arguably having sign language as your first language is a completely different thing. Since no one could first be born deaf and live her life that way, and then be born hearing and compare these experiences, I think we must conclude that we cannot know

181 whether being born deaf means that you are simply differently abled or rather disabled. If 182 being deaf does not detract from one's wellbeing in any way, the previously discussed 183 procreative principles would not require of us to produce hearing individuals when we have a 184 choice in the matter. However, deaf dogs do not have an entire unique language or a culture 185 of their own (even if they might become better at reading body language as a result of their 186 deafness). Deaf dogs are therefore disabled, and worse off than hearing dogs everything else 187 being equal (although they may still have lives very well worth living). Finally, most breeding 188 programs that are motivated by a concern for canine wellbeing deals with conditions that 189 cause physical pain and/or negative emotional states such as fear and frustration; that being in 190 pain or fear is not merely being in a different state, but a worse state, is fairly uncontroversial. 191 The same thing can be said about the second argument against any attempts of preventing 192 disabled people from being born; it is too quickly dismissed by many philosophers, but it is at 
least less relevant for dog breeding. Although attempting to create abled rather than disabled people does not logically imply that one sees disabled people as less valuable than abled ones, such an attempt might still lead disabled people to feel like failures or abled people to view the disabled in this light, and it might still lead to less resources being put into adjusting the environment to accommodate for disabilities. Social and psychological mechanisms are different from logical implications. We can see something similar in the case of dogs; people sometimes contrast breeding for fearlessness with banning fireworks, for instance, as if one could not pursue both paths simultaneously in order to reduce canine suffering as much as possible. Some people also speak, not just of breeders who habitually produce mentally and physically ill dogs, but of the dogs themselves, with contempt. I suspect that a person with this attitude, who discovered that her own dog suffered physical and/or mental problems, might treat the dog badly because of this contempt. Still, the dogs themselves cannot contemplate whether they are "failures" from a breeding standpoint, and I think problematic human attitudes can more easily be overcome in the case of dogs. It is easier to think rationally about a different species than one's own.

In the following, I will assume that those dogs that still inevitably will be born with physical or mental problems (no breeding program will be perfectly successful) will not be worse off because we strive to breed for as much canine wellbeing as possible.

\section{Pure-breds and maximizing well-being}

If we ought to breed for as much wellbeing as possible, it follows that it is not morally permissible to choose any breed one wants to when deciding to become a dog breeder. It is simply not plausible to claim that the duty to breed for wellbeing is, so to speak, only activated after the choice of breed has already and irrevocably been made. And it is true of many breeders who already do the best they can within their breed of choice that they could produce more wellbeing by switching to a different breed.

Dog breeders who struggle to improve very sickly breeds will probably find this line of reasoning uncomfortable. One might of course argue that breed preservation is important; important enough to outweigh the canine wellbeing-related reasons breeders have to switch to healthier breeds. Still, many people would be uncomfortable with the implication that if they do continue trying to improve an unhealthy breed rather than switching to an already healthy one, they would thereby sacrifice wellbeing for the sake of breed preservation. Breeders who seriously struggle to improve the health of a very sickly breed typically do not see themselves as sacrificing canine wellbeing; rather the opposite. 
227 Is there then no possible justification for BCM to be found in the philosophical literature? Are there no counter arguments to the thesis that we ought to maximize wellbeing other than those from disability advocates, already dismissed as being of little relevance for dogs?

According to David Wasserman, procreation is right when at least some of the reasons one has for creating a certain individual are concerned with her own good and the prospect of respectful relationships (Wasserman, 2005, conclusion). This means that it can be morally right to deliberately create an individual with some kind of disability. Wasserman writes

It is not clear that this evolving understanding of the family would condemn the

I do not think that Wasserman ever considered this line of reasoning to be applicable to any other species than ours (he discusses the purpose of having families in connection with reasons for having children), but it seems to me that the reasons Wasserman gives for deliberately selecting a mentally handicapped child might be brought up by a dog breeder who wants to defend her choice of a sickly and/or mentally unstable breed. She might say that although this breed has its problems, she finds breeding them and living with them especially rewarding - they have unique personalities or traits that one does not find in other dogs. Actually, I do not think this is an unusual explanation of why one chooses to breed a very sickly breed. However, I doubt that one can find a case where it is true that the dogs of a very sickly breed really have a unique personality. It is hard to see how a breed unique personality could develop unless the breeders actually select for a unique personality when choosing breeding animals by, say, using a mentality assessment test on prospective breeding animals and only let these animals reproduce who have particular, different from all other breeds, test results. Alternatively a unique personality might develop within a breed if this and only this breed is bred for performing a particular task that requires a particular personality. However, one would be hard pressed to find a breed with lots of health problems where breeding animals are selected for their ability to perform a particular task that no other breed is used for. 
It might still be the case that the breeder has a special fondness for these dogs; perhaps she just loves the breed. But now this case is disanalogous to Wasserman's; being uniquely fond of a certain look, or a certain breed history, is profoundly different from wanting a mentally disabled child because one thinks this child might have a "rare capacity for sustained, unalloyed joy".

It is also worth noting that Wasserman is in part concerned with how we ought to react to people who knowingly bring disabled individuals into the world, as he suggests that if it was wrong of the couple in his example to choose to have a mentally handicapped child, "they are subject only to a gentler, less personal reproach". The same might of course be true of all breeders who do the best they can within their breed of choice, although they could have produced more wellbeing if they had switched breed. If they do the best they can to produce dogs with a high level of wellbeing within their breed of choice they are not insensitive to canine suffering, and ought not to be harshly blamed. However, they can still be misguided, they can still be wrong in their estimation of what they ought to do.

So far, then, I have argued that given the goal of canine wellbeing, dog breeders ought to produce dogs with as much wellbeing as possible. This means not just that they ought to do the best they can within their breed of choice, but also that they ought to choose healthy over unhealthy breeds.

\section{Genetic diversity}

Only of a fairly small percentage of all dog breeders would it be true that they could not produce dogs with more wellbeing if they looked outside their breed of choice. A principle according to which we ought to produce as much canine wellbeing as possible seems to imply that lots and lots of breeds ought to be completely abandoned, from some breeds certain families or individuals ought to be picked for breeding, for only a small number of breeds ought more than a few dogs be allowed to reproduce, and in a tiny number of breeds most individuals ought to be bred on. This might seem counter intuitive to many. Is it really the case that we ought to, from a canine wellbeing perspective, abandon most breeds? This would undoubtedly be a hard bullet to bite for dog enthusiasts.

Someone might wonder at this point, whether breeders ought to abandon all breeds and stick to mutts instead - but this is probably not the optimal choice if we want to produce as much wellbeing as possible, and I will explain why.

Sweden is unique in the dog world in several respects. Over $60 \%$ of all dogs are purebreds 
The insurance companies providing health insurance for dogs record how often dogs of each

294 breed, as well as mutts, have to use their insurance. Many breeds have to use their insurance more often than mutts, indicating that they have worse health, many other breeds use it as often as mutts, and some breeds use their insurance less often (confirmed in personal correspondence with representatives for the insurance companies Sveland and Agria). This is not surprising if we consider that although mutts rarely suffer from exaggerated looks or inbreeding, they rarely reap the benefits of health examinations and selection against health problems either. It seems that we ought to focus on the healthiest breeds then, rather than mutts. There is, of course, no way of knowing whether the situation is similar in countries where this kind of statistics do not exist, but since health testing for certain illnesses before breeding is done by conscientious breeders in many countries, it is not far-fetched to suppose that many countries have a small number of purebred populations that are generally healthier than mutts. Furthermore, canine wellbeing depends on more than just physical health; mental health is often just as important. Since mutts rarely go through mentality assessment tests, we do not know how prone they are to, for instance, fear of sudden loud noises or other mental problems that diminish a dog's quality of life. It is plausible that the mentally healthiest dogs will be found in populations that either regularly go through mentality assessments of some kind or are bred to perform certain tasks that require mental stability.

It would thus seem as if we ought to abandon almost all purebreds and all mutts and focus all our breeding efforts on a tiny population of purebred dogs that have shown themselves to be optimal when it comes to wellbeing. As I said, I merely discuss pro tanto 'ought':s in this article; it might be the case that what we all things considered ought to do is something different. If we suppose that society needs herding dogs, this might justify breeding border collies (the best and therefore most popular herding dog today), even if it would turn out that they are not completely optimal from a wellbeing standpoint. But this does not take us very far. The need for herding dogs does not justify continued breeding on all breeds with a herding origin such as the other collies, corgis, pumis, Shetland sheepdog and so on. The only way to justify the preservation of many breeds seems to be to appeal to flimsier human interests (such as our interest in conformation shows). But things are not quite as they seem.

There is actually a dog-related reason for the preservation of more than a few breeds. This is the fact that an abandonment of all but a few breeds would severely deplete the canine gene pool. This might, in the long run, result in more health problems, and must therefore be weighed against the more short-term wellbeing improvements we would get if all breeders 
breeds, dog breeders would end up facing the same problems as people attempting to rescue species threatened by extinction by breeding them in zoos. Even if we start with healthy individuals, and even if we eventually build up a good-sized population from the breeding animals we began with, the lack of genetic diversity can lead to serious problems in the long run (see, for instance, Nicholas, 1996, p 241). The optimal breeding for wellbeing would thus have to balance direct selection for wellbeing-related factors such as physical and mental health against the preservation of genetic diversity.

One way to combine these two considerations might be to first determine as well as we can through empirical means at which point the negative effects of reduced genetic diversity starts to outweigh the positive effects of selection against physical and mental problems. If we assume that this can be done (probably unrealistically, but maybe we can find some very rough estimate), we can then set a threshold for mental and physical problems based on this information, cut individuals below the threshold off from breeding, but allow all individuals above it to mate with each other. There are, however, reasons stemming from canine wellbeing against doing this. The enormous diversity, mental as well as physical, within the canine species, means that it is possible for two individuals who are perfectly fine themselves to produce suffering offspring when their traits are combined in unfortunate ways. For instance, imagine a non-aggressive dog with lightning speed reactions mating with a calm, slow dog who has a good deal of aggressiveness inside her, although it takes a lot of provocation for it to show. Since mental traits are affected by lots of different genes (as well as environmental factors), most puppies will probably end up medium aggressive as well as having medium reaction speed. However, if we do many crossings of this kind, it is probable that some puppies end up having lightning speed reactions and lots of aggressiveness. (This might seem like a problem for the people around the dog rather than a canine wellbeing problem; but I think you cannot completely separate the two, since a dog can only thrive in a good home where she is wanted.) Furthermore, there is the problem of extreme physical diversity in the species. Although no actual research on this matter has been conducted to my knowledge, it is plausible that two very different body types when combined could sometimes result in health problems that neither parent suffered from. For instance, a longish body may be more problematic for a big and heavy dog than a small dog. Unless we have reason to believe that all kinds of body types can be combined with no adverse effects, we ought to adhere to a principle of caution and prevent dogs with great physical differences from breeding. 
Instead we might combine a threshold with continued pure breeding. Breeds that, on average, fall below a certain wellbeing threshold are abandoned, while we try to improve the wellbeing of the breeds above the threshold as much as possible by pursuing health programs within each breed. However, the importance of genetic diversity cannot justify anything like the status quo. That there is genetic diversity in the entire species does not benefit the dogs as long as said species is divided into small sub-populations that are kept strictly separated.

A radical suggestion for genetic diversity is to combine a wellbeing threshold with a loosening up of the nowadays mostly watertight genetic barriers between different breeds. Suppose that breed A and breed B are similar enough to be bred; the resulting offspring will be well balanced physically as well as mentally. The same holds for breed B and breed C, C and D and D and E. Now, suppose that breeders were allowed to mate dogs from each of these pairs of breeds. Breed A and breed E might be fairly different, and breeding an A-dog to an E-dog might therefore be a bad idea, but that would not be allowed either. Genetic material would still slowly drift from A to E and the other way around; with this kind of policy, the canine species would really make use of its genetic diversity. In short, for every breed, a small number of breeds considered to be close enough mentally and physically would be eligible for cross breeding at the breeders' discretion, and there would be overlap between the groups. (Allowing crossing in a manner that allows for genetic material to slowly drift from one end of the species to the other may or may not be combined with a complete fusion of very similar breeds.)

With such a loosening of the breed barriers, health programs could be made stricter than they are today. In some national kennel clubs certain breed clubs have health programs stating that, for instance, puppies cannot be registered in the national kennel club unless the parents are proven free from hip and elbow dysplasia. In breeds where deformed joints are common, however, the rules will be more lax, since strict rules would mean that too few animals could reproduce and inbreeding would ensue. The same goes for health problems that are not part of an enforced health program, as well as mental problems; in breeds where the problems are widespread, breeders will be more tolerant of breeding animals with problems. Looser barriers between breeds will thus not only increase genetic diversity but also make possible stricter health programs and health policies regarding physical as well as mental health. ${ }^{3}$ More genetic diversity and stricter health programs will result in more wellbeing.

${ }^{3}$ Obviously, there is a limit to how strict a health program realistically can be. Extremely strict programs would require vast amounts of expensive testing of breeding animals and their 


\section{Conflicts between wellbeing and breed preservation, conformation} shows and the production of working dogs

393 Now that we have seen what we ought to do given the goal of canine wellbeing, what are the

394 conflicts between this and other goals that breeders want to accomplish? Firstly, and most 395 obviously, there is a conflict between pursuing canine wellbeing and the preservation of all 396 dog breeds. From a wellbeing standpoint, we ought to abandon dog breeds. How many breeds 397 we ought to abandon depends on where the optimal balance between selection for wellbeing 398 and genetic diversity lies, but my guess is that we ought to abandon more than just a handful 399 of the very sickest breeds there are - perhaps a fairly large percentage of all breeds ought to 400 go extinct.

401 However, it is important to note that I am now talking about a 'breed' as a population of 402 dogs registered under the same breed name in the kennel clubs. In breeds, in this sense of the 403 word, where the vast majority of dogs suffer one or several problems that we know diminish 404 the dog's quality of life, and where the remainder of the dogs have close family suffering 405 from these problems (and thus almost certainly carry many genes for several serious 406 problems), the individuals of this breed ought to stop reproducing. But sometimes breed talk 407 refers more to a type of dog, recognizable by its looks and/or used for performing a certain 408 task. This is often how one uses the word 'breed' when one argues that it is important to 409 preserve various dog breeds since they are part of an ancient and important cultural heritage. 410 When someone says of a certain breed that it is several hundreds or even thousands of years 411 old, she must refer to a type of dog, since kennel clubs and therefore populations registered 412 under a common breed name in kennel clubs have existed for less than two hundred years. 413 Even if the dogs of a certain breed, in the sense of population of dogs registered under the 414 same breed name, ought not to reproduce, we might still be able to find healthy dogs of a 415 similar type and "reboot" that breed from new genetic material. If we understand 'breed' as a 416 type of dog then, the conflict between breed preservation and canine wellbeing is diminished.

417 There might still be something of a conflict here, if certain breeds-as-types are inherently 418 unhealthy. It is clear that the beauty ideals propagated at conformation shows for certain 419 breeds are unhealthy, but this is often explained away as conformation judges making bad

relatives, and the result might be that almost no dog is considered good enough. But it is, for instance, not unrealistic to breed only on dogs who are free from hip and elbow dysplasia; not if the breed barriers are loosened up. 
interpretations of the breed standards. However, it seems to me that some breed standards actually do describe unhealthy looks, and in some cases also mentalities that could be detrimental to wellbeing. Still, one might argue that we do not even have pro tanto reasons to preserve dogs according to the breed standards; our pro tanto breed preservation reasons merely concern the preservation of old dog types (since only these can be considered a valuable cultural heritage), and old dog types are never particularly extreme. I will not delve deeper into how much of a conflict there might be between wellbeing and breed preservation when breeds are considered to be types of dogs rather than particular canine populations in this article.

Is there a conflict between the goals of producing good working dogs and/or conformation show winners and wellbeing? In general, I do not think breeding for wellbeing will conflict with breeding for good working dogs, since working dogs generally work better if they are physically healthy, not excessively easily-scared and so on. It happens today that breeders prefer to breed a dog with, for instance, moderate hip dysplasia and a good working mentality over a physically healthy dog with worse personality traits, reasoning that it is better to have a dog that works hard for some years and then might have to be euthanized than a dog that never works hard since she lacks the right motivation or talent. However, such a breeder would still prefer a dog with both physical health and working capacity, and with loosened up barriers between breeds, such dogs will almost always be available. I therefore think there is not much of a conflict between the goal of producing good working dogs and wellbeing. ${ }^{4}$

When it comes to conformation shows, one might think that there is obviously a big problem here. Firstly, there are unhealthy beauty ideals at conformation shows, and secondly, there is research showing a correlation between breeding for conformation and mental problems in dogs (Svartberg, 2003, p 23). However, there are also breeds with sound beauty ideals as well as breeds bred mainly for conformation shows with a generally healthy mentality, as can be seen in the statistics for the Swedish Working Dog Association's mentality assessment test. These facts show that breeding for conformation shows is not inevitably detrimental to the wellbeing of the dogs. Why some breeds get more and more exaggerated looks while others have stayed the same during the years, and some even have

\footnotetext{
${ }^{4}$ Remember that I am only talking about breeding here; when it comes to training methods
} there might still be a conflict, since it might be the case that benevolent training methods require more resources, better trainers and/or take more time than crueller methods. 
moved from exaggerated to healthier looks during the last decades or century while the

450 breeders have continuously bred for conformation shows, would certainly be interesting to investigate, as would be the question of why some breeds but not other equally conformationbred ones have widespread mental problems. Such investigation is probably necessary if we are going to combine conformation shows with breeding for wellbeing in the future, but lies outside the scope of this article.

After all these arguments pertaining to what dog breeders ought to do, we may ask ourselves if my suggestions have any hope of being adopted by dog breeders in the real world. I will leave puppy mills outside of the discussion, since I think such breeders have little to no interest in canine wellbeing, and the only way to change their praxis is probably through legislation. However, many obstacles stand in the way of truly breeding for canine wellbeing even if we restrict our attention to small-scale breeders. As I wrote in the beginning of the article, many small-scale breeders refuse to believe that, for instance, deformed joints, extremely short muzzles or an excessively easily-scared personality cause any suffering in their dogs. And even breeders who do acknowledge these problems often regard the genetic barriers between different breeds as more or less sacred. However, small-scale breeders from Sweden as well as the USA and the UK have often (not always, but often) accepted my arguments as valid when I have laid them out the way I have in this article. Kennel clubs are big and conservative organisations, and some level of pessimism is warranted, but one ought not to conclude that turning my suggestions into praxis is down-right impossible.

\section{Conclusion}

Most breeders today think that they have pro tanto reasons to strive for wellbeing within their chosen breed, but they also think that it is morally permissible to choose any breed they like. I have argued that this belief cannot be justified. We do indeed have reason to strive for wellbeing, but this implies that we have reason to switch to another breed when doing so will produce more wellbeing in future canine generations. However, this does not imply that we ought to abandon almost all dog breeds and focus all our breeding efforts on a very small number of breeds, namely those that are optimal wellbeing-wise. Doing so would severely deplete the canine gene pool. Instead, the short-term wellbeing improvements that can be made by selecting for physical and mental health must be weighed against the long-term value of genetic diversity. For maximum wellbeing in future generations, we ought to abandon the 
481

482

483

484

485

486

487

488

489

490

491

492

493

494

495

496

497

498

499

500

501

502

506

507

508

509

503 Svartberg, Kenth. 2003. Personality in Dogs. Stockholm University (doctoral dissertation).

504 The Swedish Board of Agriculture (no author given). 20120-04-29.

505 http://www.jordbruksverket.se/amnesomraden/djur/hundarochkatter/avel.4.207049b811dd8a5

510 parents." Ethics. $116(1)$

511 Woodward, James. 1986. “The Non-Identity Problem”. Ethics. 96:804-31

worst breeds while loosening the genetic barriers between remaining breeds, and tighten up the physical and mental health programs within them.

\section{Literature}

Buchanan, Allen, Brock, David W, Daniels, Norman and Winkler, Daniel. 2000. From

Chance to Choice. Genetics and Justice. Cambridge: Cambridge University Press.

Edwards, S D. 2001. "Prevention of Disability on Grounds of Suffering." Journal of Medical Ethics. Vol 27, No 6.

Harman, Elizabeth. 2004. "Can We Harm and Benefit in Creating?" Philosophical Perspectives. Vol 18, Ethics.

Haynes, Richard. 2008. Animal Welfare. Competing Conceptions and their Ethical Implications. Springer.

Magnus Reindal, Solveig. "Disability, Gene Therapy and Eugenics: A Challenge to John Harris." Journal of Medical Ethics. Vol 26, No 2.

Nicholas, F. W. 1996. Introduction to Veterinary Genetics. Oxford: Oxford University Press. Savulescu, Julian. 2001. "Procreative Beneficence: Why we should select the best children." Bioethics. Volume 15, no 5-6.

97 Savulescu, Julian and Bostrom, Nick. Ed. 2009. Human Enhancement. Oxford: Oxford

University Press

99 Statistiska Centralbyrån, SCB (the Central Bureau of Statistics). Leading researcher: Daniel 00 Persson. 2012.

1 http://www.jordbruksverket.se/download/18.300b18bd13d103e79ef80002651/Resultatrapport

$02+$ Hundar+katter+och+andra + s\%C3\%A4llskapsdjur+2012.pdf

$06 \quad 13 \mathrm{dc} 8000462 . \mathrm{html}$

07 The Swedish Kennel Club (no author given). 2012-03-14. Ethical guidelines.

08 http://www.skk.se/uppfodning/regler-policys-och-lagar/skks-etiska-riktlinjer/ Wasserman,

09 David. 2005. "The nonidentity problem, disability, and the role morality of prospective 